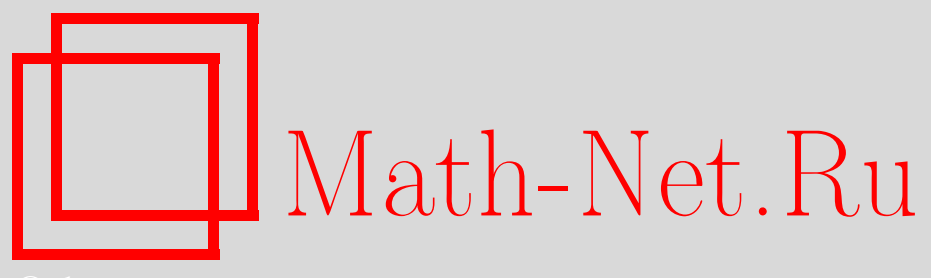

Л. А. Бокуть, Е. С. Голод, Р. И. Григорчук, В. Н. Желябин, В. Г. Кац, А. Р. Кемер, В. В. Кириченко, П. С. Колесников, С. С. Кутателадзе, В. Н. Латышев, Ю. Н. Мальцев, Г. А. Маргулис, А. В. Михалёв, А. Г. Мясников, С. П. Новиков, А. Ю. Ольшанский, А. Н. Паршин, В. П. Платонов, Ю. Г. Решетняк, Н. С. Романовский, И. А. Тайманов, О. Г. Харлампович, В. К. Харченко, Л. Н. Шеврин, И. П. Шестаков, А. В. Яковлев, Ефиму Исааковичу Зельманову 60 лет, УМН, 2016, том 71, выпуск 4, 193-199

DOI: https://doi.org/10.4213/rm9732

Использование Общероссийского математического портала Math-Net.Ru подразумевает, что вы прочитали и согласны с пользовательским соглашением http://www . mathnet.ru/rus/agreement

Параметры загрузки:

IP : 3.85 .183 .62

26 апреля 2023 г., 13:52:03

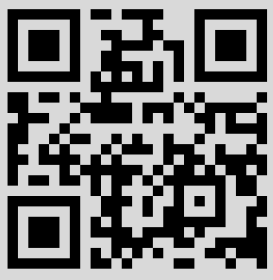




\section{Ефиму Исааковичу Зельманову 60 лет}

В 2015 г. исполнилось шестьдесят лет со дня рождения выдающегося математика, крупнейшего специалиста в современной алгебре Ефима Исааковича Зельманова.

Ефим Исаакович родился 7 сентября 1955 г. в Хабаровске, а менее чем через год семья переехала в Новосибирск. Семья была самой обычной: отец Исаак Владимирович - инженер железнодорожного транспорта, мать Нина Семеновна - библиотекарь в средней школе.

В 1972 г. Е. И. Зельманов поступил на механико-математический факультет Новосибирского государственного университета. Интересно отметить, что он был принят на первый курс "кандидатом", т.е. с условием успешной сдачи первой сессии. Условие это было им выполнено, и в 1977 г. он успешно оканчивает университет и поступает в аспирантуру. Диссертацию на соискание степени кандидата физико-математи-

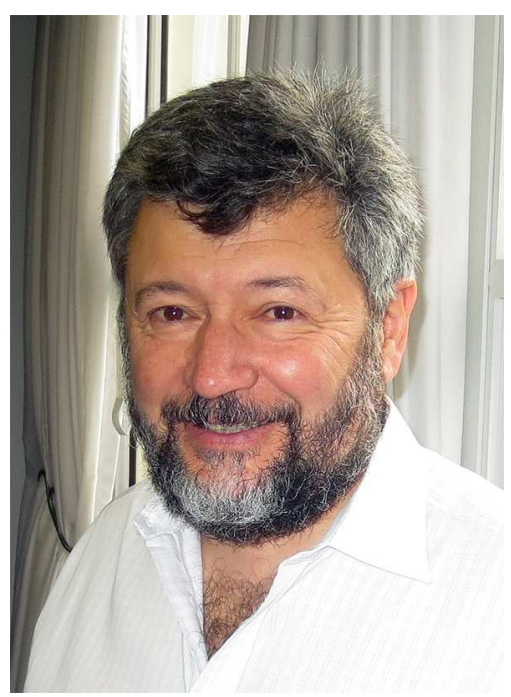
ческих наук Е. И. Зельманов защищает в 1981 г. в Новосибирске под руководством А. И. Ширшова (к сожалению, скончавшегося до успешной защиты) и Л. А. Бокутя, а степень доктора наук получает в 1985 г. в Ленинграде.

Научная деятельность Е. И. Зельманова естественно разбивается на два периода: его учеба и работа в Новосибирске (НГУ, Институт математики Сибирского отделения АН СССР) в 1972-1990 гг. и работа в университетах США (Мэдисон, Чикаго, Йель, Сан-Диего) с 1991 г. по настоящее время.

Первый можно без преувеличения назвать периодом "бури и натиска". Работая в благоприятной обстановке школы А.И. Ширшова, насыщенной новыми идеями в теории колец, особенно неассоциативных, он быстро раскрыл свои способности. Основные достижения Е. И. Зельманова этого периода можно коротко сформулировать следующим образом: он совершил то, что в школе Н. Джекобсона назвали "русской революцией в йордановых алгебрах" ${ }^{\prime}$, сделал решающий вклад в решение комбинаторных проблем Энгеля для бесконечномерных алгебр Ли, а затем развил и применил эти результаты для решения знаменитой ослабленной проблемы Бернсайда (ОБП). В своих исследованиях Ефим Исаакович "стоял на плечах гигантов" - В. Магнуса, Г. Цассенхауза, И. Н. Санова, Г. Хигмана, Ф. Холла, Н. Джекобсона, А. И. Ширшова, П. С. Новикова. Менее известно, что, штурмуя ОПБ, Ефим Исаакович был уверен в правильности своего пути и в положительном решении этой проблемы ввиду революционной для своего времени теоремы Ширшова о высоте для ассоциативных алгебр с тождествами (PI-алгебр). Дело в том, что из теоремы Ширшова следовало

\footnotetext{
${ }^{1}$ K. McCrimmon, A taste of Jordan algebras, Springer, New York, 2004, xxvi +562 p.

DOI: $10.4213 / \mathrm{rm} 9732$
} 
решение проблемы Куроша для ниль-PІ-алгебр в следующей усиленной форме: конечно порожденная PI-алгебра нильпотентна, если нильпотентны все слова некоторой ограниченной длины от порождающих. В ходе решения ОПБ возникла аналогичная проблема для алгебр Ли с той разницей, что тождества были не любые, а специального вида - линеаризованные тождества Энгеля (и нильпотентность слова Ли также следовало понимать в смысле Энгеля). Показательно, что, когда Ефим Исаакович доложил свой результат в Йеле (сразу после исторической Мальцевской конференции в Новосибирске 1989 г., где он впервые объявил о решении ОПБ), знаменитый специалист по теории групп У. Файт пошутил: "Теперь понятно, почему Вы решили ОПБ: Вы знали теорему Ширшова, а мы - нет!". В этом была доля правды: алгебраическое образование Ефима Исааковича позволяло ему использовать тонкие методы теории колец, что совсем не характерно для многих специалистов в теории групп. Изложению своих работ по йордановым алгебрам, алгебрам Ли и ОПБ Е. И. Зельманов посвятил 10 работ, опубликованных в журналах "Алгебра и логика", "Сибирский математический журнал", "Математический сборник", "Известия АН СССР", и две диссертации: "Йордановы алгебры с делением" (кандидатская диссертация, неудачная защита в ИМ СО АН СССР в октябре 1980 г., еще при жизни А.И. Ширшова; та же диссертация была успешно защищена в мае 1981 г. после вмешательства С. Л. Соболева) и "Йордановы системы и градуированные алгебры Ли" (докторская диссертация, защищена в Ленинградском университете в 1985 г.). Кроме того, эти результаты были доложены на всесоюзных алгебраических конференциях (1977, 1979, 1981), Международной конференции памяти А. И. Мальцева (1989) и международных математических конгрессах в Варшаве (1983), Киото (1990) и Цюрихе (1994).

Блестящие достижения Е. И. Зельманова послужили основанием для его приглашения в западные университеты. С 1990 по 1992 г. он работает в Оксфордском университете в Великобритании, с 1992 по 1994 г. - в Висконсинском университете в Мэдисоне (США). Затем - Чикагский университет, откуда вскоре перешел на должность профессора Йельского университета, где работал с 1995 по 2002 г. С 2002 г. по настоящее время Е. И. Зельманов работает в Калифорнийском университете в Сан-Диего.

Перечислим основные результаты Е. И. Зельманова новосибирского периода.

1. Завершено описание невырожденных (т. е. полупростых в смысле радикала Джекобсона) йордановых алгебр с условием минимальности [1]. Для них Н. Джекобсон ранее (1966) доказал структурные теоремы типа Молина-Веддерберна-Артина, но оставались открытыми две основные проблемы: описать йордановы алгебры с делением (проблема Джекобсона) и доказать нильпотентность радикала Джекобсона йордановой алгебры с условием минимальности. Обе эти проблемы были решены Е. И. Зельмановым [2].

2. Дано описание всех простых бесконечномерных йордановых алгебр [6]. Из этого результата вытекает решение фундаментальной проблемы Йордана-фон Неймана-Вигнера: существуют ли неклассические простые йордановы алгебры? Классическими называются все простые йордановы алгебры, известные на момент постановки проблемы: йордановы алгебры, возникающие из простых ассоциативных алгебр, йордановы алгебры симметрической билинейной формы и исключительные алгебры Алберта. Из результатов Е. И. Зельманова следует, что все простые йордановы алгебры - классические.

Отметим, что один новый класс неассоциативных алгебр был введен С. П. Новиковым в 1985 г. в связи с различными задачами математической физики. Отвечая на вопрос Новикова об описании конечномерных простых алгебр из этого класса над полем характеристики нуль, Е. И. Зельманов доказал, что таких алгебр не существует (точнее, все такие алгебры тривиальны) [9].

3. Структурная теория йордановых алгебр, развитая Е. И. Зельмановым, получила максимально полное расширение на класс первичных алгебр, йордановых пар 
и йордановых тройных систем [5], [8]. Были описаны невырожденные первичные йордановы алгебры (это широкий и важный класс алгебр): Е. И. Зельманов доказал, что всякая первичная невырожденная йорданова алгебра либо специальна (и такие описаны), либо изоморфна кольцу Алберта. Была решена проблема Куроша для алгебраических йордановых алгебр ограниченного индекса: все они локально конечномерны (в частности, йордановы ниль-алгебры ограниченного индекса локально нильпотентны [3]). Ранее А. И. Ширшов получил этот результат для специальных йордановых ниль-алгебр. Е. И. Зельмановым решена проблема Куроша (в форме Капланского) для йордановых алгебр с тождественными соотношениями. Йордановы пары и йордановы тройные системы - это классы алгебраических систем, тесно связанные с градуированными алгебрами Ли. Е. И. Зельманов получил полную классификацию простых йордановых пар и тройных систем. Такая общность результатов поражает воображение.

4. Получены важные продвижения в изучении свободных йордановых алгебр [4]. В частности, доказано, что эти алгебры от более чем двух порождающих имеют делители нуля, что дало ответ на соответствующий вопрос Н. Джекобсона.

5. Существенно продвинута теория $\mathbb{Z}$-градуированных алгебр Ли с конечной градуировкой. Е.И. Зельманов подошел к изучению этих алгебр Ли с точки зрения йордановой теории (рассматривая их как более общий объект по сравнению с йордановыми парами и йордановыми тройными системами). В результате применения йордановых методов им была доказана замечательная теорема о классификации всех простых $\mathbb{Z}$-градуированных алгебр Ли с конечной градуировкой над полем характеристики нуль (или достаточно большой). А именно, эти алгебры либо получаются из коммутанта простой $\mathbb{Z}$-градуированной ассоциативной алгебры или из коммутанта кососимметрической части такой алгебры относительно некоторой инволюции факторизацией по центру, либо являются конструкцией Кантора-Кехера-Титса из йордановой алгебры симметрической билинейной формы, либо совпадают с алгеброй одного из картановских типов $E_{6}, E_{7}, E_{8}, F_{4}, G_{2}, D_{4}[7]$.

6. Положительно решена ослабленная проблема Бернсайда.

Упомянутые выше ранние работы Е.И. Зельманова [1]-[8] сами по себе обеспечили ему место в ряду первых алгебраистов ХХ столетия, но решение ослабленной проблемы Бернсайда является наиболее известным в самых широких кругах математиков достижением. Этот результат был отмечен самой престижной научной наградой ХХ в. в области математики - Филдсовской премией, которую Е. И. Зельманов получил на Международном математическом конгрессе в Цюрихе в 1994 г.

История решения серии задач, известных как проблемы Бернсайда, заслуживает более подробного рассмотрения, поскольку наглядно показывает преемственность математических идей и внутреннее единство математики как рациональной науки. Кроме того, будучи чрезвычайно простой в своей формулировке, но требующей глубоких знаний и тонкой техники даже для частичного решения, данная проблема послужила для теории групп и смежных с ней областей таким же стимулом к развитию, каким для теории колец и алгебраической теории чисел была Большая теорема Ферма.

В 1902 г. Уильям Бернсайд сформулировал следующий вопрос, известный как nроблема Бернсайда: является ли конечной конечно порожденная группа, в которой порядки элементов равномерно ограничены? Для ответа на этот вопрос достаточно для любой пары натуральных чисел $d, n \geqslant 2$ рассмотреть группу $B(d, n)$, порожденную множеством из $d$ элементов, с определяющими соотношениями $x^{n}=1$ для всех слов $x$ от данного множества порождающих и выяснить, является ли эта группа конечной. В ряде частных случаев ответ был найден еще в начале ХХ столетия: для $n=3$ конечность группы $B(d, n)$ при всех $d$ установил сам Бернсайд, для $n=4-$ И. Н. Санов, а для $n=6$ - М. Холл. Но до сих пор мало что известно даже о группе $B(2,5)$. 
Поскольку до 30-х годов XX столетия не произошло существенных сдвигов в решении проблемы Бернсайда, была сформулирована так называемая ослабленная проблема Бернсайда, которая состоит в следующем: существует ли для данных натуральных чисел $d$ и $n$ максимальная (по количеству элементов) конечная $d$-порожденная группа, в которой порядки элементов ограничены константой $n$ ? Название "Restricted Burnside problem" исходит от В. Магнуса (1950). Положительное решение ослабленной проблемы Бернсайда эквивалентно тому, что группа $B(d, n)$, сама не обязательно конечная, имеет единственный максимальный конечный гомоморфный образ. Для $n=5$ ослабленную проблему Бернсайда решил положительно Г. Хигман в 1956 г.

Проблема Бернсайда была решена отрицательно: “первым звонком" стал пример Е. С. Голода (1964) бесконечной группы, в которой каждый элемент имеет конечный (но не равномерно ограниченный) порядок. ${ }^{2}$ В 1968 г. П. С. Новиков и С.И. Адян показали, что группа $B(d, n)$ бесконечна для достаточно больших нечетных $n .^{3}$

В работе Ф. Холла и Г. Хигмана 1956 г. было показано, что для положительного решения ослабленной проблемы Бернсайда достаточно решить ее для показателей $n=p^{k}$ ( $p$ - простое число) при условии, что верна гипотеза Шрайера о разрешимости групп внешних автоморфизмов простых конечных групп. (Заметим, что справедливость гипотезы Шрайера вытекает из классификации простых конечных групп.)

С другой стороны, еще в 1950 г. В. Магнус показал, что для простых показателей $(n=p)$ решение ослабленной проблемы Бернсайда сводится к обобщению классической теоремы Энгеля для алгебр Ли: для положительного решения ослабленной проблемы Бернсайда достаточно выяснить, является ли всякая алгебра Ли с тождеством Энгеля степени $p$ над полем характеристики $p$ локально нильпотентной. Положительный ответ на этот вопрос дал А. И. Кострикин $(1959,1979)$, решив тем самым ослабленную проблему Бернсайда для групп простого показателя. ${ }^{4}$

К тому моменту, когда Е. И. Зельманов приступил к работе над ослабленной проблемой Бернсайда, перед ним стояло две основные проблемы. Во-первых, обобщить результат Магнуса, сведя проблему для $n=p^{k}$ к задаче нильпотентности некоторой алгебры Ли. Это было сделано им в 1989 г. [11]. Здесь ключевую роль сыграла алгебра Ли $L(G)$, построенная по нижнему центральному $p$-ряду конечной группы $G$ показателя $p^{k}$ (этот ряд получен "измельчением" обычного нижнего центрального ряда). Алгебра $L(G)$ порождена конечным числом элементов, удовлетворяет линеаризованному тождеству Энгеля (Г. Хигман, 1958), и все (лиевы) слова от порождающих нильпотентны в смысле Энгеля (И. Н. Санов, 1951).

${ }^{2}$ См. Е. С. Голод, "О ниль-алгебрах и финитно-аппроксимируемых $p$-группах", Изв. $А H$ СССР. Сер. матем., 28:2 (1964), 273-277. - Прим. ред.

3"Первым звонком" явилась сенсационная работа П. С. Новикова 1959 г. "О периодических группах", в которой он сформулировал программу отрицательного решения знаменитой проблемы Бернсайда (см. Докл. АН CCCP, 127 (1959), 749-752). Некоторые алгебраисты не поверили в эту программу. Однако П. С. Новиков совместно с С. И. Адяном полностью реализовали эту программу в фундаментальной работе в 300 страниц (см.: П. С. Новиков, С. И. Адян, "О бесконечных периодических группах. I-III", Изв. АН СССР. Сер. матем., 32:1 (1968), 212-244; 32:2, 251-524; 32:3, 709-731). - Прим. редколлегии.

4 Этот результат (см.: А. И. Кострикин, "О проблеме Бернсайда", Изв. АН CCCP. Сер. матем., 23:1 (1959), 3-34) явился предметом докторской диссертации А.И. Кострикина (1959). Крупнейший алгебраист А.И. Мальцев на защите диссертации подчеркнул, что эта работа не проверена и что он надеется дожить до момента, когда это будет сделано. А.И. Мальцев не дожил до этого момента (он скончался в 1967 г.). Уже после смерти А. И. Мальцева семинар знаменитого алгебраиста Ивасавы нашел, что результат А. И. Кострикина не доказан. Много лет никаких публикаций на эту тему не было. С 1979 г. начинается новый период, завершившийся полным, свободным от ошибок и пробелов доказательством приведенным в книге А. И. Кострикина "Вокруг Бернсайда" (Наука, М., 1986, 232 с.). Ряд крупных алгебраистов считали, что здесь имело место идейное влияние Е. И. Зельманова. Прим. редколлегии. 
Во-вторых, следовало решить главную проблему: доказать локальную нильпотентность алгебры Ли с перечисленными выше свойствами. Важность решения этой задачи выходит далеко за пределы комбинаторной теории групп и отражает фундаментальные закономерности алгебры, проявляющиеся всюду, где используются алгебры Ли, - в геометрии, топологии, математической физике и других областях. Е. И. Зельманов полностью решил эту задачу в двух работах [13], [14], покрывающих весь спектр значений $n=p^{k}$ для всех простых $p$ и натуральных $k$. Наилучшее представление о характере этих работ дают слова А. Шалева ${ }^{5}$ : "Его потрясающее доказательство ... сочетает в себе удивительную техничность с чрезвычайно оригинальными идеями из различных дисциплин. Доказательство использует глубокую структурную теорию (квадратичных) йордановых алгебр, ранее разработанную Маккриммоном и Зельмановым ${ }^{6}$, а также теорию разделенных степеней и другие инструменты; он также опирается на совместную работу Кострикина и Зельманова ${ }^{7}$, в которой устанавлена локальная нильпотентность так называемой сэндвич-алгебры. В то время как алгебры Ли уже давно считаются естественной рабочей площадкой в контексте ограниченной проблемы Бернсайда, появление йордановых алгебр является беспрецедентным и весьма удивительным".

Из положительного решения ослабленной проблемы Бернсайда вытекает решение другой известной проблемы о топологических группах, сфомулированной В. П. Платоновым: всякая периодическая компактная группа является локально конечной [15].

Охарактеризуем теперь наиболее известные результаты Е. И. Зельманова зарубежного периода.

7. Совместно с Дж. Бенкарт был полностью решен вопрос описания бесконечномерных алгебр Ли, градуированных системой корней. Эта задача была впервые рассмотрена С. Берманом и Р. Муди (1992), ими же были описаны такие алгебры для корневых систем типов $A, D, E$ с точностью до центральной изогении (адекватной в данном контексте эквивалентности, связанной с изоморфизмом центральных расширений). В работе [17] задача была решена для всех типов корневых систем. Примечательно, что для систем типов $B, C, G$ и $F$ ключ к формулировке и доказательству классификационной теоремы был найден в теории йордановых алгебр.

8. В соавторстве с М. Расином [21] и К. Мартинес [19] завершено построение структурной теории конечномерных йордановых супералгебр положительной характеристики. В случае характеристики нуль строение таких супералгебр было ранее описано В. Кацем (с дополнением И. Кантора). Кроме того, в ряде совместных работ с К. Мартинес и И. П. Шестаковым [22]-[24] Е. И. Зельманов описал все неприводимые представления этих супералгебр; данное направление работ также можно считать завершенным с появлением работы [25]. Очень крупным достижением Е. И. Зельманова в соавторстве с K. Мартинес и В. Кацем является классификация бесконечномерных $\mathbb{Z}$-градуированных йордановых супералгебр с равномерно ограниченными размерностями однородных компонент [20].

9. Доказана гипотеза Дж. Вильсона (1991) о том, что про-р-группа, удовлетворяющая условию Голода (это достаточное условие бесконечности про- $p$-группы, которое Е. С. Голод использовал для построения своего знаменитого примера), содержит свободную неабелеву про- $p$-группу. Частичное подтверждение этой гипотезы получили Дж. Вильсон и Е. И. Зельманов [16], доказав, что условие Голода влечет существование "обычной" свободной подгруппы; полностью гипотеза была доказана в [18].

${ }^{5}$ J. Lindenstrauss, L. C. Evans, A. Douady, A. Shalev, N. Pippenger, "Fields medals and Nevanlinna prize presented at ICM-94 in Zürich", Notices Amer. Math. Soc. 41:9 (1994), $1103-1111$.

${ }^{6}$ Имеется в виду работа [10].

${ }^{7}$ Работа [12]. 
Мы упомянули только наиболее известные работы Ефима Исааковича, а общее число его научных статей превышает 100. Он успешно воспитывает молодых исследователей по алгебре. К настоящему времени у Ефима Исааковича более 10 прямых учеников.

Трудно переоценить то влияние, которое научный авторитет и личное обаяние Ефима Исааковича оказывают на развитие алгебры в разных странах. Он ведет активную научно-организаторскую работу, координируя, в частности, научную деятельность таких организаций, как Корейский институт высших исследований (Сеул, Республика Корея) и Университет короля Абдул-Азиза (Джидда, Саудовская Аравия), где он занимает должность Distinguished Professor. Он состоит в ряде профессиональных объединений: в Национальной академии наук США, Американском математическом обществе (почетный член), Американской академии наук и искусств, Королевской академии Испании, Европейской академии наук, Корейской академии науки и технологии, Бразильской академии наук. Ряд университетов Европы и Америки удостоили его звания почетного доктора. В их числе Университет Квинс, Университет Алберты, Университет Овьедо, Университет Сент-Андруса, Алтайский, Киевский и Хагенский университеты.

Мы, друзья и коллеги Ефима Исааковича, желаем ему дальнейших научных достижений, крепкого здоровья и счастья.

Л. А. Бокуть, Е.С. Голод, Р. И. Григорчук, В.Н. Желябин, В.Г. Каи, А.Р. Кемер, В.В. Кириченко, П. С. Колесников, С. С. Кутателадзе, В.Н. Латышев, Ю.Н. Мальцев, Г.А. Маргулис, А.В. Михалев, А.Г. Мясников, С.П. Новиков, А.Ю. Ольшанский, А. Н. Паршин, В. П. Платонов, Ю. Г. Решетняк, Н. С. Романовский, И. А. Тайманов, О.Г. Харлампович, В.К. Харченко, Л. Н. Шеврин, И. П. Шестаков, А. В. Яковлев

\section{Цитированные работы Е. И. Зельманова}

[1] “Йордановы алгебры с условиями конечности", Алгебра и логика, 17:6 (1978), 693-704; англ. пер.: "Jordan algebras with finiteness conditions", Algebra and Logic, 17:6 (1978), 450-457.

[2] "Йордановы алгебры с делением", Алгебра и логика, 18:3 (1979), 286-310; англ. пер.: "Jordan division algebras", Algebra and Logic, 18:3 (1979), 175-190.

[3] "Йордановы ниль-алгебры ограниченного индекса", Докл. АН CCCP, 249:1 (1979), 30-33; англ. пер.: "Jordan nil algebras of bounded index", Soviet Math. Dokl., 20:6 (1979), 1188-1192.

[4] "О первичных йордановых алгебрах", Алгебра и логика, 18:2 (1979), 162-175; англ. пер.: "Primary Jordan algebras", Algebra and Logic, 18:2 (1979), 103-111.

[5] "О первичных йордановых тройных системах", Сиб. матем. журн., 24:4 (1983), 23-37; англ. пер.: "Prime Jordan triple systems", Sib. Math. J., 24:4 (1983), 509-520.

[6] "О первичных йордановых тройных системах. II", Сиб. матем. журн., 25:5 (1984), 50-61; англ. пер.: "Primary Jordan triple systems. II", Sib. Math. J., 25:5 (1984), 726-735.

[7] “Алгебры Ли с конечной градуировкой”, Матем. сб., 124(166):3(7) (1984), 353-392; англ. пер.: "Lie algebras with a finite grading", Math. USSR-Sb., 52:2 (1985), 347-385.

[8] "О первичных йордановых тройных системах. III", Сиб. матем. журн., 26:1 (1985), 71-82; англ. пер.: "Primary Jordan triple systems. III", Sib. Math. J., 26:1 (1985), 55-64. 
[9] "Об одном классе локальных трансляционно инвариантных алгебр Ли", Докл. AH CCCP, 292:6 (1987), 1294-1297; англ. пер.: "On a class of local translation invariant Lie algebras", Soviet Math. Dokl., 35 (1987), 216-218.

[10] "The structure of strongly prime quadratic Jordan algebras", Adv. in Math., 69:2 (1988), 133-222 (with K. McCrimmon).

[11] "О некоторых проблемах теории групп и алгебр Ли", Матем. сб., 180:2 (1989), 159-167; англ. пер.: "On some problems of group theory and Lie algebras", Math. USSR-Sb., 66:1 (1990), 159-168.

[12] "Теорема о сэндвичевых алгебрах", Теория Галуа, кольиа, алгебраические групnы и ux приложения, Сборник статей, Тр. МИАН СССР, 183, Наука. Ленинградское отд., Л., 1990, 106-111 (совм. с А.И. Кострикиным); англ. пер.: "A theorem on sandwich algebras", Proc. Steklov Inst. Math., 4 (1991), 121-126 (with A. I. Kostrikin).

[13] "Решение ослабленной проблемы Бернсайда для групп нечетного показателя", Изв. АН СССР. Сер. матем., 54:1 (1990), 42-59; англ. пер.: "Solution of the restricted Burnside problem for groups of odd exponent", Math. USSR-Izv., 36:1 (1991), 41-60.

[14] "Решение ослабленной проблемы Бернсайда для 2-групп", Матем. сб., 182:4 (1991), 568-592; англ. пер.: "A solution of the restricted Burnside problem for 2-groups", Math. USSR-Sb., 72:2 (1992), 543-565.

[15] "On periodic compact groups", Israel J. Math., 77:1-2 (1992), 83-95.

[16] "Identities for Lie algebras of pro-p groups", J. Pure Appl. Algebra, 81:1 (1992), 103-109 (with J. S. Wilson).

[17] "Lie algebras graded by finite root systems and intersection matrix algebras", Invent. Math., 126:1 (1996), 1-45 (with G. Benkart).

[18] "On groups satisfying the Golod-Shafarevich condition", New horizons in pro-p groups, Progr. Math., 184, Birkhäuser Boston, Boston, MA, 2000, 223-232.

[19] "Simple finite-dimensional Jordan superalgebras of prime characteristic", J. Algebra, 236:2 (2001), 575-629 (with C. Martinez).

[20] Graded simple Jordan superalgebras of growth one, Mem. Amer. Math. Soc., 150, № 711, Amer. Math. Soc., Providence, RI, 2001, x+140 pp. (with V. G. Kac, C. Martínez).

[21] "Simple Jordan superalgebras with semisimple even part", J. Algebra, 270:2 (2003), 374-444 (with M. L. Racine).

[22] "Jordan superalgebras and their representations", Algebras, representations and applications, Contemp. Math., 483, Amer. Math. Soc., Providence, RI, 2009, 179-194 (with C. Martínez).

[23] "Representation theory of Jordan superalgebras. I", Trans. Amer. Math. Soc., 362:2 (2010), 815-846 (with C. Martínez).

[24] "Jordan bimodules over the superalgebras $P(n)$ and $Q(n)$ ", Trans. Amer. Math. Soc., 362:4 (2010), 2037-2051 (with C. Martínez, I. Shestakov).

[25] "Irreducible representations of the exceptional Cheng-Kac superalgebra", Trans. Amer. Math. Soc., 366:11 (2014), 5853-5876 (with C. Martínez). 